Full Title: $\quad$ Cross-cultural psychiatry and validity in DSM-5

Short title: Cross-cultural psychiatry and validity

Authors (i.e. name as it should appear on the paper):

\title{
Tim Thornton
}

Position (i.e. job description as it should appear on the paper):

Tim Thornton

Professor of Philosophy and Mental Health

Address for correspondence:

School of Health

University of Central Lancashire

Preston

PR1 2HE

Other Contact Details:

phone 01772894646

fax

email:

TThornton1@uclan.ac.uk

Key-words (not appearing in title):

DSM-5, cultural concepts of disorder, culture, khyal cap, taxonomy,

Word count (excluding title page but including references):

$\sim 6,400$

Character count (abstract):

Software used: Microsoft Word

Filename(s): 


\section{Cross-cultural psychiatry and validity in DSM-5}

\section{Abstract}

The latest edition of the American Psychiatric Association's Diagnostic and Statistical Manual, DSM-5 explicitly addresses the possibility of cultural variation in mental illness. Among other things, it contains a 'Glossary of Cultural Concepts of Distress' which describes nine common conditions including khyal cap, or 'wind attacks,' a syndrome found among Cambodians in the United States and Cambodia. The chapter examines three possible models of the relation between such cultural idioms and the ambition, for DSM-5, of being a valid taxonomy of universal forms of mental illness. The models are: 1) an underlying universal 'pathogenic' component overlain by a variable 'pathoplastic' cultural shape. 2) a pathogenic-only model. 3) a pathoplastic-only model. None, however, reconciles the DSM's simultaneous ambitions of validity and cultural sensitivity and suggest that the 'Glossary of Cultural Concepts of Distress' remains an afterthought in tension with the rest of the taxonomy.

\section{Introduction}

The fifth edition of the American Psychiatric Association's Diagnostic and Statistical Manual, DSM-5, puts greater emphasis than previous editions on cross-cultural factors affecting mental illness [APA 2013]. Diagnostic criteria have been revised to take account of cross-cultural variation, there is a more specific cultural formulation and a glossary lists nine 'Cultural Concepts of Distress'. But the DSM does not present a clear view of whether it assumes that mental illnesses are universal and apply across all cultures or whether they can be specific and local to particular cultures. Nor does it give an account of the relation of the cultural concepts to the rest of the taxonomy of disorders or the extent to which they are put forward as valid diagnoses.

The first section of this chapter outlines the ways that cultural factors have been included in Diagnostic and Statistical Manual DSM-5 [APA, 2013]. It introduces one particular example: khyal cap or wind attacks, a syndrome found among people living in Cambodia. The second section sets out three possible views of the nature of such cultural concepts of distress. On one view, which dates back to the German psychiatrist Karl Birnbaum, an underlying universal 'pathogenic' component is overlain by a variable 'pathoplastic' cultural shape [Birnbaum 1923]. This combination suggests the conceptual possibility of two single factor models in which either factor is set to zero: pathogenic-only and pathoplastic-only. The final section returns to the example of khyal cap. On inspection, none of the three models helps accommodate its own incompatible aetiological theory with the biomedical view of the rest of the DSM. This suggests that the very idea of cultural concepts of distress fits uneasily with the aspirations to validity of the rest of DSM-5.

\section{Cultural factors in DSM-5}

The fourth edition of the Diagnostic and Statistical Manual DSM-IV introduced guidelines for a 'cultural formulation' and a 'Glossary of Culture-Bound Syndromes' [APA, 1994]. The cultural formulation 'supplement[ed] the nomothetic or standardized diagnostic ratings with an idiographic statement, emphasizing the patient's personal experience and the corresponding cultural reference group' [Mezzich et al., 1999; P459]. The 'culture-bound syndromes' were described as 'localityspecific patterns of aberrant behavior and troubling experience that may or may not be linked to a particular DSM-IV diagnostic category' [APA, 2000; P898].

These new elements in the DSM reflected, firstly, awareness of the need for it to address growing cultural diversity within North America since '[i]mmigrants bring with them their own indigenous patterns and conceptions of mental illness, some of which are structured into cultural syndromes' [Guarnaccia and Rogler, 1999: 1322]. Secondly, the DSM needed to contain cross-cultural material because of its increasing global use. 
DSM-5 offers a more extended treatment of cultural factors in psychiatric diagnosis than DSM-IV [APA, 2013]. Throughout the manual, diagnostic criteria have been revised to reflect cross-cultural variations in presentations of disorders. The discussion of the 'Cultural Formulation' in section III now sets out a semi-structured interview. In the Appendix, there is a 'Glossary of Cultural Concepts of Distress' which describes nine common conditions. The Introduction warns how cultural factors might affect diagnosis and prognosis and thus should be investigated in a cultural formulation [APA, 2013; P14]. The manual suggests that culture can affect any of the following:

- The boundaries between normality and pathology for different types of behaviour.

- Vulnerability and suffering (for example, by amplifying fears that maintain panic disorder).

- The stigma of, or the support for, mental illness.

- The availability of coping strategies.

- The acceptance or rejection of a diagnosis and treatments, affecting the course of illness and recovery.

- The conduct of the clinical encounter itself and how this affects the accuracy of diagnosis, acceptance of treatment, prognosis and clinical outcomes.

The Introduction to DSM-5 also summarises (in fact at greater length than the later discussion of the cultural formulation in the main text) three distinct ways that culture can impact on diagnoses [APA, 2013]. The single idea of culture-bound syndromes from DSM-IV is replaced by three notions: cultural syndromes, cultural idioms of distress and cultural explanations (or perceived causes) of illnesses (or symptoms). It is worth quoting the summary in full:

1. Cultural syndrome is a cluster or group of co-occurring, relatively invariant symptoms found in a specific cultural group, community, or context (e.g. ataque de nervios). The syndrome may or may not be recognized as an illness within the culture (e.g. it might be labelled in various ways), but such cultural patterns of distress and features of illness may nevertheless be recognizable by an outside observer.

2. Cultural idiom of distress is a linguistic term, phrase, or way of talking about suffering among individuals of a cultural group (e.g. similar ethnicity and religion) referring to shared concepts of pathology and ways of expressing, communicating, or naming essential features of distress (e.g. kufiingisisa). An idiom of distress need not be associated with specific symptoms, syndromes, or perceived causes. It may be used to convey a wide range of discomfort, including everyday experiences, subclinical conditions, or suffering due to social circumstances rather than mental disorders. For example, most cultures have common bodily idioms of distress used to express a wide range of suffering and concerns.

3. Cultural explanation or perceived cause is a label, attribution, or feature of an explanatory model that provides a culturally conceived etiology or cause for symptoms, illness, or distress (e.g. maladi moun). Causal explanations may be salient features of folk classifications of disease used by laypersons or healers. [ibid: P14]

Although the authors distinguish between these different ideas, they concede that the same elements may play a role in all three categories. For example, depression is used as an idiom of distress whether of: 1) an illness or pathology, or 2) normal but significant sadness. It is also recognised as a mental illness syndrome gathering together a number of symptoms. Finally, it is taken to be the cause of those symptoms. Just as depression can play the role of syndrome, idiom of distress and explanation, so can other concepts local to other cultures.

Given this complication, although the 'Glossary of Cultural Concepts of Distress' describes nine common cultural syndromes, the concepts described may also play a role as idioms of distress and purported explanations or causes of experiences. The 'Cultural Concepts of Distress' described are khyal attacks or khyal cap, ataque de nervios ('attack of nerves'), dhat ('semen loss'), kufungisisa ('thinking too much'), maladi moun ('humanly caused illness') nervios ('nerves'), shenjing shuairuo ('weakness of the nervous system'), susto ('fright'), taijin kyofusho ('interpersonal fear disorder'). 
Each is related to similar but different concepts found in other cultures and also to the illness categories set out in the main body of DSM-5. Khyal cap, for example, is linked to panic disorder.

In the years leading up the publication of DSM-5, much emphasis was placed on the attempt to increase the validity of psychiatric diagnostic categories. The book A Research Agenda for DSM-V which comprises a series of papers on different aspects of DSM- 5 starts with this thought [Kupfer et al 2002].

Those of us who have worked for several decades to improve the reliability of our diagnostic criteria are now searching for new approaches to an understanding of etiological and pathophysiological mechanisms - an understanding that can improve the validity of our diagnoses and the consequent power of our preventive and treatment interventions. [Kupfer et al 2002: Pxv]

This passage expresses the worry that whilst work had been done to improve the reliability of DSMIII and DSM-IV, not enough attention had been paid to the validity of the syndromes within psychiatric taxonomy. This aim for the rest of DSM-5 suggests the following question about the 'Glossary of Cultural Concepts of Distress': What is its relation to the rest of the taxonomy, to the other diagnostic categories set out in the main body of the book? There seem to be three general possibilities. 1) The status of the glossary and the main body might be intended to the same and both aim at validity: describing real universal mental illness categories. 2) The status of both could be intended to be the same and all diagnostic categories be thought to be culturally specific. 3) The appendix might be intended to have a distinct lesser status, not aimed at validity but rather charting the theoretical errors of other cultures.

The second option is the most conceptually fraught. It requires, not just that the rates or prevalence of an illness that can apply universally varies between cultures but rather that the validity of a diagnosis, the very idea of an illness, is in some sense true only of or for a particular culture. The paradox of such relativism is that it is unclear that one can assert its general truth. But asserting its merely relative truth does not seem enough. To adopt this view of the main body of the DSM is to adopt a self-consciously ironic or relativist view. One possibility - obviously not taken in DSM-5 - is that some diagnoses from the main section belong in the appendix. Perhaps anorexia nervosa is somehow specific to European and North American cultures whilst schizophrenia is universal. So can the aim of cultural sensitivity exemplified in the Cultural Formulation and the articulation of cultural idioms of distress go hand in hand with the scientific ambitions of twenty-first century psychiatry? Or are the two ventures somehow in tension?

These general questions can be illustrated through one example. One of the nine items in the 'Glossary of Cultural Concepts of Distress' is khyal cap which is described thus.

\section{Khyal cap}

'Khyal attacks' (khyal cap), or 'wind attacks,' is a syndrome found among Cambodians in the United States and Cambodia. Common symptoms include those of panic attacks, such as dizziness, palpitations, shortness of breath, and cold extremities, as well as other symptoms of anxiety and autonomic arousal (e.g., tinnitus and neck soreness). Khyal attacks include catastrophic cognitions centered on the concern that khyal (a windlike substance) may rise in the body-along with blood-and cause a range of serious effects (e.g., compressing the lungs to cause shortness of breath and asphyxia; entering the cranium to cause tinnitus, dizziness, blurry vision, and a fatal syncope). Khyal attacks may occur without warning, but are frequently brought about by triggers such as worrisome thoughts, standing up (i.e., orthostasis), specific odors with negative associations, and agoraphobic type cues like going to crowded spaces or riding in a car. Khyal attacks usually meet panic attack criteria and may shape the experience of other anxiety and trauma- and stress or related disorders. Khyal attacks may be associated with considerable disability.

Related conditions in other cultural contexts: Laos (pen lom), Tibet (srog rlunggi nad), Sri 
Lanka (vata), and Korea (hwa byung).

Related conditions in DSM-5: Panic attack, panic disorder, generalized anxiety disorder, agoraphobia, posttraumatic stress disorder, illness anxiety disorder. [ibid: P834]

The belief that illness can be caused by a dysfunction of a wind-like substance, described using the same word as for wind, seems to be common in parts of Asia [Hinton et al., 2010; P245]. Khyal is thought normally to flow alongside the blood supply and can pass out of the body through the skin. But the flow can become disturbed 'surging upward in the body toward the head, often accompanied by blood, to cause many symptoms and possibly various bodily disasters' [ibid: P245]. It is thought to be caused by, for example, 'worry, standing up, a change in the weather and any kind of fright, such as being startled or awakening from a nightmare [ibid: P246]. Local treatments include dragging a coin along the skin giving rise to characteristic abrasions.

To 'coin,' the person dabs the tip of a finger in khyal ointment (preing kenlaa), a Vaseline-like substance containing camphor and menthol, and then drags the fingertip along the skin to create a streak 5 or 6 inches in length. Next a coin is grasped by the fingers and the edge pushed down slightly against the skin at the proximal beginning of the streak; the coin is then dragged outward along the streak of khyal ointment. This is then repeated. [ibid: P271]

Despite the overlap of symptoms, it is clear that the framework of beliefs that surround the conception of khyal attack differs from that of biomedical psychiatry. What then is its supposed status in DSM-5? This question calls for a general understanding of the ways in which culture might affect concepts of illness and whether any model can simultaneously aim for validity whilst admitting cultural variation. Thus the next section will outline three general ways of thinking about the cultural dependence of mental illness categories, the possible role of cultural formulation and hence the different cultural concepts of distress in DSM- 5 .

\section{Three models of cultural concepts of distress}

\section{A two-factor pathogenic-pathoplastic model}

One way to understand how culture affects mental illness would be to think of the expression of mental illness as the result of two-factors: an invariant endogenous factor and a local cultural appearance: 'pathogenic' versus 'pathoplastic' factors [Birnbaum 1923]. The psychiatrist and anthropologist Roland Littlewood stresses the connection between this distinction and the longstanding distinction in psychiatry between the form and content of mental illness.

To deal with variations in the symptoms between individuals, while maintaining the idea of a uniform disease, clinical psychiatry still makes a distinction between the essential pathogenic determinants of a mental disorder - those biological processes which are held to be necessary and sufficient to cause it - and the pathoplastic personal and cultural variations in the pattern. Those two are still distinguished in everyday clinical practice by the particularly nineteenth century German distinction between form and content. [Littlewood, 2002: P5]

This distinction needs handling with some care. Littlewood suggests that the pathogenic factor is a necessary and sufficient cause of mental disorder. But the notion of cause suggests a state distinct from the mental disorder it causes. Further, the requirement of a sufficient cause is difficult to attain as causes are only sufficient relative to an assumed causal field [Mackie, 1993]. The connection to the distinction of form and content suggests a better interpretation is not what causes mental disorder but what constitutes it. The pathogenic factor is then the set of essential properties of disorders, the properties that are necessary and sufficient for a state to count as a disorder. The pathoplastic factor is the contingent variation of inessential properties of the disorder.

Littlewood reports that in the biomedical view of psychiatry the pathogenic factor is a biological process. In other words, the essential features of mental disorder can be described in biological 
terms. Such a view fits an influential analysis of mental disorder in general articulated and defended by Jerome Wakefield [Wakefield, 1999]. According to Wakefield, a disorder is a harmful dysfunction, where function and hence dysfunction is picked out in accordance with evolutionary theory. Evolutionary theory specifies the biological functions of the traits of the human mind and body. Note that the focus on social dysfunction in the DSM is not the same as biological dysfunction. In fact, it better accords with Wakefield's invocation of harm. But central to his attempt to offer a unified account of both mental and physical illness, biological functions include evolutionarily selected mental functions, both cognitive and affective. On this model, the essential or pathogenic properties of a disorder can be described not just in biological terms but, more specifically, as biological dysfunctions explicated through evolutionary theory. (The mental character of the biological dysfunctions which constitute mental disorders will be discussed shortly.) In the case of illnesses where there remains ignorance of biological mechanisms, the idea of a pathogenic factor is an article of faith: a commitment to there being some universal underlying nature to the illness in question.

Although biomedical psychiatry favours a biological characterisation of pathogenic factors, other candidates are possible. Consider Louis Sass' account of Schreber's delusions in Paradoxes of Delusion [Sass, 1994]. Schreber was a German judge diagnosed with dementia praecox, now classed as schizophrenia, who wrote a first person account of his illness, including his delusions, called Memoirs of My Nervous IIIness, at the start of the twentieth century. Sass attempts to shed light on the nature of Schreber's delusions by comparing them to philosophical solipsism.

Solipsism is the view that the only thing that exists in the world is the self of the person who thinks about it. It is expressed in the necessarily first person thought: 'Only I and my mental states exist'. Everything else is merely an idea (for me: one of ' $m y$ ideas'). Solipsism is thus a form of idealism according to which only ideas exist - taken to the logical limit. If everything that exists is merely an idea only the first person subject of thought (for me: ' $l$ ') can have those ideas. So only one person exists. This paradoxical thought is used by Sass to shed light on the paradoxical quality of schizophrenic delusions.

[Schreber's] mode of experience is strikingly reminiscent of the philosophical doctrine of solipsism, according to which the whole of reality, including the external world and other persons, is but a representation appearing to a single, individual self, namely, the self of the philosopher who holds the doctrine... Many of the details, complexities, and contradictions of Schreber's delusional world... can be understood in the light of solipsism. [ibid: P8]

The elucidation or understanding that Sass seeks isn't merely aimed at one particular delusional experience or even at all of Schreber's experiences considered as a whole. It is meant to shed light more generally on the nature of schizophrenia itself. The reason it can (according to Sass) is that the experiences that characterise schizophrenia derive from a general and abstract feature of rationality:

[Madness] is, to be sure, a self-deceiving condition, but one that is generated from within rationality itself rather than by the loss of rationality. [ibid: P12]

Although a general feature of rationality, Sass himself does not think that the failure within rationality that amounts to schizophrenia is culturally universal. Rather, he thinks that is the result specifically of modernism [Sass, 1992]. But if, contra Sass, solipsism were not merely the product of recent European culture but rather a standing universal possibility suggested by the abstract structure of rationality itself, then its corresponding disorder - schizophrenia - would be a risk for any rational subject, human or alien, whatever their biology or evolutionary history. The pathogenic factor is, on this model, an abstract feature of rationality rather than a particular biological process or dysfunction.

Whether the pathogenic factor is thought of as a biological or a more abstract feature of rationality, on the pathogenic-pathoplastic model variation in general and cultural variation specifically (the focus here) enters with the pathoplastic factor. Culturally invariant pathologies of underlying human 
nature are overlaid by local cultural variation in how they are expressed. 'Expressed' could carry either of two meanings. First, it might mean that standing possibilities for biological dysfunction or failings of rational subjectivity might be differently prompted or caused by different social or geographical contexts. The idea that mental illness has social determinants is, however, akin to socially caused variation in heart disease rates in different cultures and hardly merits the label 'cultural concept'.

The more interesting idea is that variation in 'expression' picks out the way in which underlying pathologies might be plastic to the different self-interpretations that people in different cultures possess and thus the way the pathologies are experienced and avowed. This would be an example of a cultural idiom of distress in the vocabulary of the DSM-5. But whereas for physical illness, how one understands one's illness might be thought to be an accidental superficiality compared with the real underlying condition (as understood, perhaps, by the medical profession), one might argue that for mental illness its esse is percipi: how it is perceived at least partly constitutes it.

On a two-factor pathogenic-pathoplastic model, mental illnesses either are, or are underpinned by, pathologies of some sort of universal substrate. The difference between these options is the difference between thinking that the alloy of an invariant underlying pathology and a varying cultural overlay itself comprises what we mean by a mental illness, and on the other hand thinking that the mental illness proper is identical with the pathogenic factor only.

One might think, for example, that khyal cap and panic disorder have the same underlying biological mechanism but that the characteristic way in which, in the former, subjects think of their distress through the conceptual lens of a dysfunction of the flow of wind-like substance is sufficient to mark it off as a different kind of mental illness from the latter. Biological dysfunction is then the common component of two distinct illnesses depending on cultural context. Christopher Boorse' distinction between disease and illness where the latter is tied to the subject's experience of it implies a difference in illness in such a case [Boorse 1975]. On the other hand, one might think that the real illness is whatever is common to khyal cap and panic disorder: the pathogenic factor. It is merely that the appearance that the single illness takes can vary.

Whichever view is taken of whether the pathogenic factor is the illness or merely the common disease underpinning of different illnesses, a two-factor pathogenic-pathoplastic view of cultural concepts of mental illness suggests a particular view of the aim of a cultural formulation in psychiatric diagnosis. It is a way of inferring, from locally divergent symptoms, the universal underlying nature of mental illness. The aim of sensitivity to cultural difference would be to find a way to penetrate beneath it to a common substrate appropriate for scientific psychiatric research.

This seems to be the view of the ex-president of the World Psychiatric Association Juan Mezzich et al. (2009) in their discussion of 'Cultural formulation guidelines' when they say:

The cultural formulation of illness aims to summarize how the patient's illness is enacted and expressed through these representations of his or her social world. [Mezzich et al 2009; P390]

and

Performing a cultural formulation of illness requires of the clinician to translate the patient's information about self, social situation, health, and illness into a general biopsychosocial framework that the clinician uses to organize diagnostic assessment and therapeutics. In effect, the clinician seeks to map what he or she has learned about the patient's illness onto the conceptual framework of clinical psychiatry. [ibid: P391]

These passages suggest that there is a division between how an illness is enacted and expressed and the underlying biological mechanisms explored by biomedical psychiatry. The former is culturally 
shaped, the latter is invariant. On Mezzich et al.'s (2009) account, the only positive role cultural factors can then play is as a source of contingent health promoting resources:

The aim is to summarize how culturally salient themes can be used to enhance care and health promotion strategies (e.g., involvement of the patient's family, utilization of helpful cultural values). [ibid: P399]

In other words, 'culturally salient themes' do not reveal the shape of mental illnesses in themselves but can, contingently, be used to promote health because of their effects on how people understand their own illnesses. All this suggests that the underlying view of the role of cultural formulation is determined by a two-factor view. Such a view is, however, merely one of several possible. I will argue that it is a half way house between two more radical views of the possibilities for cultural psychiatry both of which are versions of a single factor which I will now outline.

\section{Two single factor models of cultural variation: pathoplastic-only and pathogenic-only}

A two-factor pathogenic-pathoplastic model of the nature of transcultural psychiatry requires a distinction between surface appearance and underlying pathology. But it might be that this distinction cannot be drawn. The various ways one might attempt to flesh out the contrast between underlying pathology - for example as biological or some other underpinning notion of universal human nature - and surface appearance might fail.

Consider the first suggestion for the pathogenic factor outlined above: a biological process which, if Wakefield is right, can be more precisely specified as a harmful dysfunction analysed using evolutionary theory. Drawing a distinction between this and the surface appearance in the way a two-factor model requires might seem unproblematic for some illnesses. It requires that a common underlying biological dysfunction can be identified despite different culturally imposed behaviours or experiences. But in the case of some mental dysfunctions there may be no principled way of drawing a distinction between an underlying dysfunction and the surface appearance.

To illustrate this, consider the role of biological processes. It seems plausible to assume mental functions and dysfunctions depend, in potentially complicated ways, on human brains. Thus there may be common biological processes underpinning common mental dysfunctions. But one cannot treat just any shared biological process as the first factor of a mental illness. The biological process has to be a mental process: a failure of a mental function. It is then much less clear how there can be shared mental dysfunctions between different manifestations. The dysfunction may be located only at the surface mental level.

The potential difficulty of dividing between underlying pathology and surface appearance can also be described without talk of mental functions. The philosopher John McDowell argues that human nature can be divided between two distinct levels: biological nature and a 'second nature' that has to be developed through education and enculturation [McDowell 1994; P183]. A good example is initiation into a first language. Whilst the ability to develop a second nature is contingent on biological first nature, biology alone is not enough. This suggests the possibility of two kinds of mental illness. So called 'organic' illnesses, such as dementia or alcohol syndromes, are those with a clear biological or first nature component. But 'functional' disorders are, on this view, disorders only of second nature. In the latter case, it is unclear how to distinguish between the surface appearance of mental illness, its characteristic experiences or manifestations, for example, and an underlying mental process. Mental illness - or at least some mental illnesses - may be features of the surface appearance of our second nature.

If the distinction between the two levels on which the two-factor pathogenic-pathoplastic model depends cannot be drawn for at least some mental illnesses that leaves only a single factor. There are, however, two possible one-factor models depending on whether one thinks of illness as all pathogenic or all pathoplastic. 
A one-factor model need not imply that there is any substantial cultural variation of mental illness beyond prevalence rates. Cultural factors might play a role in causing different rates of illness in different communities without this making the nature of illness in any sense relative to a culture. Using McDowell's vocabulary, this might be because human second nature is itself universal. Or, using Wakefield's model, it might be because the mental dysfunctions that constitute illnesses are universal. A pathogenic-only model holds that illness varies only in external features such as rates and superficial and unimportant local understandings of it. Any apparent deeper variation would be a mark of our ignorance, our misdiagnosis. So a pathogenic-only model has no need for a cultural formulation to extract or excavate the underlying commonalities because they are open to view.

But, following the account suggested in this chapter of the distinction between pathogenic and pathoplastic not in terms of the causes of mental illness but their essential and universal properties, it is possible to articulate a radical pathoplastic-only model. According to this, there might be no shared pathogenic factor between apparently different mental illnesses in different cultures. Cultural variation might go 'all the way down'. Genuinely different forms of mental illness would emerge from different ways of living in different societies. It would thus be a 'category fallacy', in Arthur Kleinman's phrase, to assume that a form of illness found in one culture must, in principle, have application in another [Kleinman, 1977].

To flesh this example out it will be helpful to consider again Sass' account of schizophrenia. The symptoms of schizophrenia are a kind of lived experience of the philosophical theory of solipsism. Sass thus claims that schizophrenic delusion is generated from within rationality itself rather than by the loss of rationality. That basic idea can be used to illustrate both the pathogenic-only and the pathoplastic-only models of cultural idioms of distress.

If one thinks that the history of Western philosophy merely illustrates and unpacks conceptual connections implicit in the rationality of any possible thinker - human or even alien - then solipsism is also a standing possibility for anyone and hence, on Sass' account, so is schizophrenia as its lived version. That is the pathogenic-only model. Any apparent culturally determined local variation in the experience of schizophrenia, such as the specific contents of delusions by contrast with invariant forms, would be merely superficial, requiring no great cultural sensitivity to detect. It is the thought that it is merely or trivially superficial which distinguishes this from a two-factor model with its demand for a cultural formulation to penetrate surface features.

If, on the other hand, one thinks with Sass himself, that Western philosophy has been driven not merely by the abstract demands of rationality but by historically contingent assumptions about the nature of mind, world and subjectivity then the temptation towards solipsism will seem to be a merely local cultural matter. At the risk of over simplifying Sass' view, if the intellectual movement of Modernism had not existed then there would have been no such thing as schizophrenia [Sass 1992]. This is a pathoplastic-only view because it implies that there need be no common elements to the mental illnesses experienced in different cultures. (This is not to say that such illness is uncaused. On the gloss offered in this chapter, pathogenic versus pathoplastic concerns what is essential and universal versus what is accidental rather than what causes mental illness.)

The pathoplastic-only model is more radical than the two-factor model even though both agree on the need for some sort of cultural formulation. A pathoplastic-only version of a cultural formulation does not enable one to dig beneath surface difference to find underlying common pathologies but would instead be an articulation of the genuinely different ways people can be ill in different cultures. According to it, there are genuinely different forms of mental illness which need have nothing substantial in common across different cultures. The virtues of the validity of a psychiatric taxonomy and its universality diverge.

In fact, sympathy for a pathoplastic-only view of mental illness sometimes seems to go hand in hand with a view that questions the illness-status of cultural idioms of distress. For example, Littlewood's anthropological comparison of female overdosing in Anglo-American society with the behavioural 
patterns of women in 'less pluralistic small-scale societies' looking 'not just at the person involved but at the local meaning of the act in the political context in which it happens' suggests a social function rather than an individual pathology [Littlewood 2002: P36]. It may be that the pathoplasticonly model requires an anthropological stance and that such a stance looks for and tends to find social order rather than individual illness or disorder. But that is not an essential feature of a pathoplastic-only approach. There is nothing inconsistent with the idea that a pathoplastic-only model is a model of illness.

\section{The status of khyal cap}

The first section of this chapter introduced but left hanging the question of whether the presence in the DSM-5 of the 'Glossary of Cultural Concepts of Distress' implied a kind of anthropological relativism or whether it is consistent with the privileging of a particular cultural standpoint: that of twenty-first century biomedical psychiatry. The example of khyal cap was used to highlight the issue since it involves a distinct view of physiology which includes the flow of a wind-like substance along the blood vessels and normally, harmless, out through the skin.

In its case, at first sight no such relativism seems necessary given the definitions of cultural syndrome, idiom of distress and explanation set out in DSM-5 [APA 2013: 14]. Khyal cap can serve as an 'idiom of distress': the conception of an experience had by a subject. If someone describes their experience as the rising up of a wind-like substance then that is simply an anthropological fact about the culture. It can serve as a 'cultural explanation' because, again, that is a fact about how a culture explains particular experiences without implicit endorsement of that theory of aetiology by the sufferer?. But, by the standards of twenty-first century western psychiatry, it can even be described as a 'cultural syndrome' since that is defined as 'a cluster or group of co-occurring, relatively invariant symptoms found in a specific cultural group, community, or context'. If, for whatever reason, the symptoms described co-occur then it is reasonable to call them ' $k$ hyal cap'. In other words, the sincere use of 'khyal cap' by a cross-cultural psychiatrist need not cause any intellectual difficulty.

But such a reading of the description carries some implications when it comes to understanding the nature of culturally sensitive psychiatry. If the concept of a khyal attack is only ever used within the context of what someone from that culture believes - his or her conception of the nature and explanation of their experiences - rather than as an objective description of what is really causing the attack, then that suggests a distinction of kind between cultural concepts of distress and the main elements of DSM-5's taxonomy.

Consider the question asked from a biomedical psychiatric standpoint: 'But from what are they really suffering?'. The description above suggests a ready answer selected from the list of related conditions in DSM-5: 'Panic attack, panic disorder, generalized anxiety disorder, agoraphobia, posttraumatic stress disorder, illness anxiety disorder'. Such a response suggests that a culturally sensitive psychiatry might be merely a sensitivity to other cultures' errors: the truthful ascription of a false belief about the causes of abnormal experiences.

With that worry in the background, consider the example of khyal attack through the range of options explored above. Recall Mezzich et al's (2009) suggestion that the role of a cultural formulation is to 'map what he or she has learned about the patient's illness onto the conceptual framework of clinical psychiatry'. This reflects a two-factor model. If applied to this case, the underlying invariant pathogenic factor is whatever is picked out by 'panic attack, panic disorder, generalized anxiety disorder, agoraphobia, posttraumatic stress disorder, [or] illness anxiety disorder'. The varying local cultural shape is the 'catastrophic cognitions centered on the concern that khyal (a windlike substance) may rise in the body'. Fitting khyal attack into the two-factor model does nothing to address the underlying worry, however, because there remains an asymmetry between it and panic attack. From the perspective of the rest of the DSM, the former, but not the 
latter, involves an error about the real aetiology of the condition. Dividing the condition between two-factors does nothing to change this perspective.

Nor does it help to adopt the pathogenic-only model. That model presents a stark choice for any putative newly discovered mental illness. Cultural syndromes such as khyal cap can have either of two statuses. They are either really other names for universal conditions also picked out by the vocabulary of biomedical psychiatry such as 'panic disorder'. Or they do not exist. For example, if it is an essential part of the theoretical apparatus of khyal cap that it is caused by the rising up of a windlike substance then given that on our best account of physiology there is no such substance then, equally, there is no such condition. Those who self-report it, or its characteristic symptoms, are in some sense in error about their own conditions. Again the underlying worry is not addressed.

Could khyal cap be understood in accord with the pathoplastic-only model? In rejecting the traditional two-factor model of cross-cultural psychiatry (then generally called 'transcultural psychiatry' [Littlewood 1986a: 38]) anthropologically minded psychiatrists such as Roland Littlewood and Arthur Kleinman have implicitly favoured a pathoplastic-only model [eg Littlewood 1985, 1986, 2002].

[C]ulture-bound syndromes are representations in the individual of symbolic themes concerning social relations and which occur in certain personal and historical situations. They articulate both personal predicament and public concerns by means of which women and other depressed categories exert mystical pressure upon their superiors in circumstances of deprivation and frustration when few other sanctions are available to them' [Littlewood 1985: 704]

Such a view plays down the illness status of the behaviour and emphasises instead its positive social function in addressing an imbalance of power. In his paper 'The culture-bound syndromes of the dominant culture', Littlewood applies the same style of analysis onto diagnoses found in the main sections of DSM. Agoraphobia, for example, is argued to serve an adaptive function for a woman against her husband without open defiance, binding them both together at home [Littlewood and Lipsedge 1986: 262-3].

So it might seem that a pathoplastic-only approach can address a worry about the asymmetric treatment of syndromes in the main body of the DSM and the appendix. If the same pathoplasticonly approach is taken to the syndromes set out in the rest of DSM-5 then it seems that those in the 'Glossary of Cultural Concepts of Distress' have the same status. But there is a cost to this. It is not that an ironic attitude to the cultural concepts is avoided. It is rather that it is generalised to include diagnoses favoured by biomedical psychiatry for example: agoraphobia, anorexia nervosa. In any case, debunking psychiatric syndromes set out in the main body of the DSM by arguing that they are really meaning-laden adaptive strategies rather than genuine pathologies may be plausible in some cases (perhaps ADHD, personality disorder, depression following bereavement). But it seems implausible across the board. A globally critical attitude to every mental illness syndrome is a high price to pay for affording cultural concepts equal status.

But, as was argued above, a pathoplastic-only approach need not deny the pathological status of conditions in favour of adaptive social functions. An anthropological investigation could be of different forms of illness. On a pathoplastic-only approach, this requires some universal concept of illness in general whilst denying that illnesses need be universal. Jerome Wakefield analysis of illness as harmful dysfunction provides one such universal standard [Wakefield 1999]. Bill Fulford argues that illness is value-laden failure of ordinary doing [Fulford 1989]. Either of these general accounts of the concept of illness could serve for an anthropological investigation of cultural variations in forms of illness. Behaviour which amounts to agoraphobia in the UK might involve no failure of ordinary doing in a culture in which a sub-population is not expected to venture outside, for example. 
Despite the possibility of a pathoplastic-only approach to local conceptions of pathology (rather than socially adaptive functions), this does not help in the case of khyal cap. The problem is that it involves not just a description of a local failure of function or action. In fact, the possibility of construing it as a variant of panic attack or disorder suggests a continuity of the kind of failure of function or action that it embodies with those recognised in the main body of the DSM. Rather, the main difference lies within the local aetiological theory. But this is not merely different from but rather incompatible with the view of the body contained within biomedical psychiatry. Espousing both a traditional biomedical view in the main body of the text and an incompatible view in the appendix threatens the validity of one or the other. They cannot both be set out as true accounts.

\section{Conclusion}

One of the criticisms of western psychiatry has been its cultural narrow mindedness, reflecting a particular socio-cultural perspective but without realising this. Littlewood, for example, argues in Pathologies of the West, that psychiatry has often assumed that experience of mental illness in America and Europe is more purely pathogenic whilst other cultures embody a kind of error: 'a poor imitation of European forms' [Littlewood 2002: 10]. The fact that DSM-5 has more explicitly addressed the nature of cultural idioms of mental distress than previous editions might suggest progress has been made in addressing this criticism.

Despite this, however, there remain tensions within the DSM in accommodating the cultural concepts of distress. As the example of khyal cap illustrates, they can be framed in local aetiological theories or local accounts of physiology which are incompatible from the views of biomedical psychiatry expressed in the main body of the text. None of the three models of the relation of disorder to culture helps.

To summarise: on a pathogenic-only view, cultural idioms of distress accord with DSM categories, correct or augment them or embody errors. But the model rules out the idea of cultural variation. The traditional two factor pathogenic-pathoplastic model allows that khyal cap may contain a genuine pathogenic core, which reflects diagnostic categories from the main body of the DSM, but the difference in physiological theory corresponding to the idea of windflow along the blood vessels is a local, pathoplastic error. Whilst it is true that those self-ascribing khyal cap are distressed it is not true that this results from dysfunction of an inner wind. The more radical pathoplastic-only model suggests the possibility of genuinely distinct conditions in virtue of different conceptions of flourishing, or ordinary actions, or societal functions (depending on the view taken of the concept of disorder). Such a view contains a variety of relativism. To be ill is relative to the practices or functions or actions of a local culture. But there is no reason to think that the truths of human physiology are in that sense relative to local cultures. And hence the pathoplastic-only model is no help in accommodating khyal cap.

This result is, perhaps, unsurprising. Although these cultural concepts of distress are flagged in the Introduction and discussed in the main body of DSM-5, their articulation and description is restricted to an appendix. They do not form a part of the taxonomy of mental illnesses proper whose aims include validity.

Discussion of the abstract models of 'Glossary of Cultural Concepts of Distress' does, however, suggest two different approaches to the relation between future psychiatric taxonomies and anthropological investigation. On a pathogenic-only or a two-factor pathogenic-pathoplastic view, a completed psychiatric taxonomy would contain a finite number of underlying universal conditions, overlain, according to the latter view by different culturally imposed appearances. But on a pathoplastic-only model there are as many possible illnesses as there are ways of being unable to take part in local ways of life or local conceptions of flourishing. This challenges the idea of universal diagnostic categories. A compendious version of the DSM would have to chart conditions that, by virtue of their local cultural origins, would not be applicable globally. Given that the arguments for or 
against any one of the different views of cultural concepts do not produce clear results, this remains a live possibility.

\section{Acknowledgement}

This chapter was written whilst a fellow of the Institute for Advanced Study, University of Durham. My thanks both to the IAS, Durham and the University of Central Lancashire for granting me research leave.

\section{Bibliography}

American Psychiatric Association (1994) Diagnostic and Statistical Manual of Mental Disorders Fourth Edition (DSM-IV), Washington, D.C.: American Psychiatric Association

American Psychiatric Association (2000) Diagnostic and Statistical Manual of Mental Disorders Fourth Edition Text Revision (DSM-IV-TR), Washington, D.C.: American Psychiatric Association

American Psychiatric Association (2013) Diagnostic and Statistical Manual of Mental Disorders Fifth Edition (DSM-5), Washington, D.C.: American Psychiatric Association

Birnbaum, K. ([1923] 1974) 'The Making of a Psychosis' Translated by H. Marshall, in S. R. Hirsch \& M Shepherd (eds) Themes and Variations in European Psychiatry, Bristol: John Wright. 197-238

Bolton D. (2008). What is mental disorder? An essay in philosophy, science and values, Oxford: Oxford University Press

Boorse, C. (1975) 'On the distinction between disease and illness' Philosophy and Public Affairs 5: 4968

Cummins, R. (1975) 'Functional Analysis' Journal of Philosophy 72: 741-65

Fulford, K.W.M. (1989) Moral Theory and Medical Practice, Cambridge: Cambridge University Press

Guarnaccia, P.J. and Rogler, L.H. (1999) 'Research on Culture-Bound Syndromes: New Directions' Am J Psychiatry 156: 1322-1327

Hinton, D.E., Pich, V., Marques, L., Nickerson, A. and Pollack, M.H. (2010) 'Khyal Attacks: A Key Idiom of Distress Among Traumatized Cambodia Refugees' Cult Med Psychiatry 34: 244-278

Kleinman, A. M. (1977). Depression, somatization and the 'new cross-cultural psychiatry'. Social Science and Medicine, 11, 3-10.

Kupfer, D. J., First, M. B. and Regier, D. A. (eds) (2002) A Research Agenda for DSM-V, Washington, DC.: American Psychiatric Association

Littlewood, R. (1985) 'The migration of culture-bound syndromes' In E. Pichot (ed) Psychiatry The State of the Art Volume 8 History of Psychiatry, National Schools, Education, and Transcultural Psychiatry, London: Springer: 703-707

Littlewood, R. (1986) Russian dolls and Chinese boxes: an anthropological approach to the implicit models of comparative psychiatry', in J. L. Cox (ed.) Transcultural Psychiatry, Beckenham: Croom Helm: 37-58

Littlewood, R. (2002) Pathologies of the West, London: Continuum

Littlewood, R. and Lipsedge, M. (1986) 'The culture-bound syndromes' of the dominant culture: culture, psychopathology and biomedicine'. In J. L. Cox (ed.) Transcultural Psychiatry, Beckenham: Croom Helm: 253-273

Mackie, J.L. (1993) 'Causes and conditionals' in Sosa, E. and Tooley, M. (eds) Causation, Oxford: Oxford University Press: 33-50 
Mezzich J.E., Kirmayer L.J., Kleinman A., et al (1999) 'The place of culture in DSM-IV' J Nerv Ment Dis 187: 457-464

Mezzich, J.E., Caracci, G., Fabrega Jr., H. and Kirmayer, L.J. (2009) 'Cultural formulation guidelines' Transcultural Psychiatry 46: 383-405

Sass, L.A. (1994) The Paradoxes of Delusion, New York: Cornell

Sass, L.A. (1992) Madness and modernism, New York: Cornell

Wakefield, J.C. (1999) 'Mental disorder as a black box essentialist concept' Journal of Abnormal Psychology 108: 465-472

Wright, L. (1973) 'Functions' Philosophical Review 82: 139-68 